\title{
Incremento, ingresso e mortalidade em uma floresta de contato ombrófila aberta/estacional em Marcelândia, Estado do Mato Grosso
}

\author{
Chirle COLPINI ${ }^{1}$, Versides Sebastião Moraes e SILVA², Thelma Shirlen SOARES ${ }^{3}$, Niro HIGUCHI ${ }^{4}$, Danielle \\ Parra TRAVAGIN ${ }^{2}$,José Vespasiano Lisboa ASSUMPÇÃO ${ }^{5}$
}

\begin{abstract}
RESUMO
Esta pesquisa teve por objetivo estudar o incremento em diâmetro, área basal e volume, o ingresso e a mortalidade de uma floresta ombrófila aberta/estacional no município de Marcelândia. Os dados são provenientes de 69 parcelas permanentes instaladas e medidas em 2001 e remedidas em 2003 e 2007. Foram avaliados o número de indivíduos e os incrementos em diâmetro, área basal e volume para o período de 2001 a 2007. O ingresso foi determinado como sendo as árvores que atingiram ou ultrapassaram o diâmetro de $17 \mathrm{~cm}$. A mortalidade foi calculada pela soma de todas as árvores com diâmetro igual ou superior a $17 \mathrm{~cm}$ encontradas mortas em cada medição. No período considerado de seis anos, teve como resultado para o incremento em diâmetro, área basal e volume respectivamente, $0,34 \mathrm{~cm} ; 0,22 \mathrm{~m}^{2} \cdot \mathrm{ha}^{-1} \mathrm{e} 2,11 \mathrm{~m}^{3} \cdot \mathrm{ha}^{-1}$. Os valores médios para as taxas de mortalidade e ingresso foram, respectivamente, $0,78 \%$ e $0,30 \%$.
\end{abstract}

PALAVRAS-CHAVE: dinâmica florestal; floresta inequiânea, floresta Amazônica.

\section{Increment, entry and mortality in a open/seasonal ombrophilous contact forest into Marcelândia, Mato Grosso State, Brazil}

\section{ABSTRACT}

This research aimed to study the increase in diameter, basal area, volume, the entry, and the mortality of an open/seasonal ombrophilous forest in the city of Marcelândia. Data were collected in 69 permanent plots established in 2001 and remeasured in 2003 and 2007. The number of individuals and the increases in diameter, basal area, and volume for the period 2001 to 2007 were evaluated. The entry was determined by the trees that have reached or exceeded a diameter of $17 \mathrm{~cm}$. Mortality was calculated as the sum of all found dead trees in each measurement with a diameter equal or more than $17 \mathrm{~cm}$. In the considered period of six years, the results to the increase in diameter, basal area, and volume were respectively, $0.34 \mathrm{~cm}, 0.22$ $\mathrm{m}^{2} \cdot \mathrm{ha}^{-1}$, and $2.11 \mathrm{~m}^{3} \cdot \mathrm{ha}^{-1}$. The average values for the mortality rates and entry respectively were $0.78 \%$ and $0.30 \%$.

KEYWORDS: Forestry Dynamics. Heterogeneous Natural Forest, Rainforest (Amazon forest).

\footnotetext{
1 Serviço Florestal Brasileiro, E-mail: chcolpini@yahoo.com.br

2 Universidade Federal de Mato Grosso, E-mail: versides@ufmt.br; danielle.parra@gmail.com

3 Universidade Federal de Goiás - Campus Jataí, E-mail: thelmasoares@terra.com.br

${ }^{4}$ Instituto Nacional de Pesquisas da Amazônia, E-mail: niro@inpa.gov.br

5 IBAMA, Escritório Regional de Juína-MT, E-mail: vespasianoassumpcao@gmail.com
} 


\section{INTRODUÇÃO}

Uma característica fundamental dos ecossistemas é a dinâmica. Este processo é o mecanismo por meio do qual a floresta se mantém em equilíbrio, mantém a sua estrutura e composição ao longo do tempo. O estado atual de um povoamento florestal é resultado da interação de vários processos em particular o crescimento, a mortalidade e a regeneração (LAMPRECHT, 1990; DAJOZ, 2006).

Fundamentalmente os processos de dinâmica de sucessão natural de florestas tropicais dependem da formação de clareiras por morte ou queda natural de árvores (JARDIM et al.,1993). As clareiras surgem em decorrência da morte, decomposição de árvores em pé e/ ou queda de árvores mortas ou vivas de grande porte. As causas mais freqüentes são a morte por envelhecimento, a incidência de raios, tombamento, quebraduras e o ataque de fungos e insetos (LAMPRECHT, 1990).

De acordo com Hosokawa et al. (1998), o estudo dos processos dinâmicos (crescimento e produção, mortalidade e ingresso) de uma floresta é de grande importância, visto que estes parâmetros indicam o crescimento e as mudanças ocorridas em sua composição e estrutura. Portanto, a prediçấo confiável destes processos, principalmente para os parâmetros crescimento e produção, torna-se imprescindível para a adoção de tratamentos e medidas silviculturais adequados ao manejo da floresta sob regime de rendimento sustentado.

No Brasil, pesquisadores como Mendonça (2003), Oliveira (2005), Azevedo (2006), D’Oliveira e Braz (2006), Teixeira et al. (2007), Braga e Rezende (2007), entre outros, estudaram a dinâmica de florestas naturais. Entretanto, no Estado de Mato Grosso, ainda há escassez de informaçôes acerca das taxas de crescimento, ingresso e mortalidade das árvores em florestas nativas, fato este que motivou o desenvolvimento deste estudo, uma vez que o estabelecimento do ciclo de corte e retiradas máximas periódicas presumidas em planos de manejo florestal sustentável só são possíveis de serem previstos por meio do conhecimento destes parâmetros.

Neste contexto, este estudo teve por objetivo determinar o incremento periódico anual em diâmetro, área basal e volume e as taxas de ingresso e mortalidade.

\section{MATERIAIS E MÉTODOS}

\section{CARACTERIZAÇÃO DA ÁREA}

A área de estudo localiza-se na Estação Experimental de Manejo Florestal "Pedro Nonato da Conceição", da Universidade Federal de Mato Grosso (UFMT) em cooperação com a Tecanorte Empreendimentos Florestais Ltda., nas coordenadas de $10^{\circ} 36^{\prime} 03^{\prime \prime}$ e $11^{\circ} 07^{\prime} 35^{\prime \prime}$ de Latitude Sul e $53^{\circ} 25^{\prime} 50^{\prime \prime}$ e $54^{\circ} 03^{\prime} 40^{\prime \prime}$ de Longitude Oeste, no Município de Marcelândia, estado do Mato Grosso.

O clima é do tipo AM de Köppen (Clima Tropical Monçóico). A temperatura média anual é de $28^{\circ} \mathrm{C}$ e a precipitação total anual varia de 2.250 a $2.550 \mathrm{~mm}$, distribuídas em duas épocas distintas durante o ano uma chuvosa que ocorre de janeiro a março e a outra, período sem chuvas, entre junho e agosto.

O solo foi classificado como Latossolos Vermelho/Amarelo distróficos, de textura média, que predominam associados à Latossolos Vermelho Escuro distróficos, de textura média e Areias Quartzosas distróficas (BRASIL, 1981).

Segundo BRASIL (1981), trata-se de uma área de tensão ecológica, na região de contato floresta ombrófila/floresta estacional, com predominância da formação denominada floresta semidecidual, submontana, dossel emergente. Nestas áreas no período de seca, embora a maioria das espécies componentes do dossel arbóreo seja de árvores tipicamente amazônicas, cerca de $20 \%$ dos indivíduos perdem pelo menos parcialmente suas folhas, o que possibilita classificá-la como floresta estacional.

\section{FONTE DE DADOS}

Os dados utilizados sáo provenientes de 69 parcelas permanentes de $10 \mathrm{mx} 250 \mathrm{~m}$ instaladas pelo método de amostragem de área fixa empregando-se o processo de amostragem sistemática em dois estágios em que as unidades amostrais foram selecionadas em duas etapas entre linhas e entre unidades na linha. A primeira amostra foi selecionada aleatóriamente e as demais distribuídas com distâncias fixas entre unidades de amostra dentro da linha e entre linhas, conforme descrito por Péllico Neto e Brena (1997).

O levantamento amostral ocorreu em três ocasióes: a primeira medição foi realizada em 2001, a segunda em 2003 e a terceira ocorreram em 2007.

Em cada parcela amostral as árvores com dap (diâmetro a 1,3 metros do solo) maior ou igual a $17 \mathrm{~cm}$ foram medidas, identificadas e plaqueteadas. As árvores que apresentaram irregularidades como sapopemas, nós, calosidades, podridáo, danos ou qualquer deformação no ponto de medição a circunferência foi tomada cerca de $20 \mathrm{~cm}$ acima dessas irregularidades.

As remediçóes das parcelas foram efetuadas sempre no período de seca e a remarcação do ponto de medição, reemplaqueteamento e checagem dos nomes científicos das espécies foram realizados nestas ocasióes.

Árvores mortas (mortalidade), bem como os indivíduos que passaram a atingir o tamanho mínimo de medição a cada ocasiáo (ingresso), foram registradas e computadas. A identificação dos indivíduos foi realizada, na floresta, por mateiros experientes da regiáo e paralelo a identificação o material botânico de 
algumas espécies não identificadas em campo foram coletadas para posterior identificação com o apoio do Herbário Central da UFMT, onde as exsicatas foram depositadas.

\section{PROCESSAMENTO E ANÁLISE DOS DADOS}

Para avaliar a dinâmica da floresta em estudo, foram efetuados os cálculos dos incrementos periódicos para diâmetro, área basal e volume conforme descritos por CHAPMAN e MEYER (1949), PRODAN (1968) e ASSMANN (1970), as taxas relativas de ingresso e mortalidade basearam-se nas prediçóes de VANCLAY (1994). Estes valores foram calculados para todas as espécies e para o conjunto de espécies comerciais para o período de 2001 a 2007.

Os ingressos ou recrutamento foram considerados como sendo o número de árvores que atingiram ou ultrapassaram o diâmetro mínimo de $17,0 \mathrm{~cm}$, em cada mediçáo, a partir da segunda. Esse valor foi dividido pelo número de anos do intervalo entre as mediçóes para obter o número de ingressos por hectare por ano. A partir desse valor foi calculada a percentagem anual de ingressos, pela relação entre o número de novos indivíduos em cada medição e o número de árvores presentes na medição anterior.

A mortalidade foi considerada como sendo o número de árvores com diâmetro maior ou igual a $17,0 \mathrm{~cm}$ encontradas mortas em cada medição, a partir da segunda. Para o cálculo da mortalidade foram considerados os seguintes estados: árvore morta em pé, árvore morta caída ou quebrada por ação de agentes naturais.

O número de árvores registradas como morta em cada mediçấo foi dividida pelo número de anos do intervalo entre os levantamentos para obter o número de mortas no período e, em seguida, expresso na unidade por hectare por ano. A partir desse valor, assim como no caso dos ingressos, foi calculada a percentagem anual de mortalidade pela relação entre o número de árvores mortas em cada medição e o total de árvores presentes na medição anterior.

Para estimar os volumes individuais das árvores foram testadas funçóes para expressar o volume a partir do diâmetro, conforme relacionado na Tabela 1.

Para o ajuste dos modelos, foram utilizados dados referentes à cubagem de 275 árvores-amostra (Tabela 2). Os diâmetros foram mensurados às alturas de $0,20 \mathrm{~m}, 1,30 \mathrm{~m}, 2,0$ $\mathrm{m}$ e a partir deste em intervalos regulares de 2,0 $\mathrm{m}$ até a base da copa. Nas árvores com presença de sapopema as medidas foram tomadas a 0,30 $\mathrm{m}$ acima destas, com intervalos regulares de 2,0 $\mathrm{m}$. Empregou-se o processo de seleção aleatória das árvoresamostra em funçáo da distribuiçáo em classes diamétricas, com amplitude da classe de $10 \mathrm{~cm}$, buscando obter árvore representante na diagonal de todas as classes de diâmetro e altura na floresta.

$\mathrm{Na}$ amostra foram consideradas todas as espécies de interesse comercial e as potenciais para madeira serrada e laminada. Os modelos foram ajustados pelo método dos mínimos quadrados descrito por Draper \& Smith (1981). Cada modelo foi avaliado pelos: coeficiente de determinaçáo ajustado, erro padrão de estimativa e análise da distribuiçáo gráfica dos resíduos. Para fins de comparação dos modelos logarítmicos com os não logarítmicos foi recalculado o erro padrão de estimativa e o coeficiente de determinação conforme recomendado por Machado et al. (2004).

A área basal por hectare foi obtida pela soma das áreas seccionais de cada espécie em cada parcela e, em seguida, expresso por hectares em função da área amostrada. Já o volume por hectare foi obtido pela somatória dos volumes individuais de cada árvore encontrada em cada parcela que, posteriormente foi dividido pela área total da amostra.

Tabela 1 - Modelos testados para estimar o volume total das árvores individuais do povoamento*.

\begin{tabular}{lcc}
\hline Modelo & Autor & Modelo \\
\hline 1 & Kopezky - Gehrhardt & $\mathrm{V}=\beta_{0}+\beta_{1} \mathrm{~d}^{2}+\varepsilon_{i}$ \\
2 & Hohenad - Krenn & $\mathrm{V}=\beta_{0}+\beta_{1} \mathrm{~d}+\beta_{2} \mathrm{~d}^{2}+\varepsilon_{i}$ \\
3 & Husch & $\ln (\mathrm{V})=\beta_{0}+\beta_{1} \ln (\mathrm{d})+\varepsilon_{i}$ \\
4 & Brenac & $\ln (\mathrm{V})=\beta_{0}+\beta_{1} \ln (\mathrm{d})+\beta_{2} \frac{1}{\mathrm{~d}}+\varepsilon_{i}$ \\
\hline 5 & Exponencial cúbica & $V=e^{\left(\beta_{0}+\beta_{1} \mathrm{~d}+\beta_{2} \mathrm{~d}^{2}+\beta_{3} d^{3}\right)}+\varepsilon_{i}$
\end{tabular}

* Em que: In = logaritmo neperiano; $v=$ volume $\left(\mathrm{m}^{3}\right) ; \mathrm{d}=$ diâmetro medido a $1,3 \mathrm{~m}$ do solo $(\mathrm{cm}) ; \beta_{0}, \beta_{1}, \beta_{2}$, e $\beta_{3}=$ parâmetros a serem estimados; $\varepsilon_{i}=$ erro aleatório 
Tabela 2 - Distribuição de freqüência das árvores-amostra da cubagem.

\begin{tabular}{|c|c|c|c|c|c|c|c|c|}
\hline \multirow{2}{*}{ Classe de dap (cm) } & \multicolumn{7}{|c|}{ Classe de altura (m) } & \multirow{2}{*}{ Tota } \\
\hline & $6-8$ & $8-10$ & $10-12$ & $12-14$ & $14-16$ & $16-18$ & $18-20$ & \\
\hline $10-20$ & 1 & 2 & 5 & 1 & 1 & 1 & & 11 \\
\hline $20-30$ & & 1 & 5 & 3 & 2 & 2 & & 13 \\
\hline $30-40$ & & 4 & 3 & & 1 & & & 8 \\
\hline $40-50$ & 1 & 2 & 8 & 1 & 1 & & & 13 \\
\hline $50-60$ & 1 & 11 & 31 & 21 & 5 & 4 & 1 & 74 \\
\hline $60-70$ & 2 & 13 & 21 & 22 & 13 & 5 & 1 & 77 \\
\hline $70-80$ & 2 & 4 & 11 & 10 & 8 & 4 & 1 & 40 \\
\hline $80-90$ & & 2 & 6 & 7 & 1 & 2 & 1 & 19 \\
\hline $90-100$ & & 1 & 5 & 4 & & 3 & & 13 \\
\hline $100-110$ & & & 1 & & 1 & & & 2 \\
\hline $110-120$ & & & & 1 & 1 & 1 & & 3 \\
\hline $120-130$ & & & 1 & & 1 & & & 2 \\
\hline Total & 7 & 40 & 97 & 70 & 35 & 22 & 4 & 275 \\
\hline
\end{tabular}

Os crescimentos em diâmetro, área basal e volume foram calculados a partir da diferença entre as medidas nas duas ocasióes e o incremento periódico anual (IPA) pela divisão do crescimento pelos anos correspondentes a cada período considerado.

\section{RESULTADOS E DISCUSSÃO}

\section{EQUAÇÃO VOLUMÉTRICA}

Após o ajuste das equações e análise dos parametros dos modelos foi selecionado o modelo que apresentou o melhor desempenho na estimativa do volume para árvores individuais baseado no maior coeficiente de determinação ajustado, no menor erro padrão da estimativa e na normalidade da distribuição dos resíduos em relação à abscissa.

A equação de Husch, $\operatorname{Ln} V=-7,56601+(2,08812 \times \operatorname{Ln}(d))$, foi a que permitiu obter estimativas mais precisas dos volumes individuais na área em estudo, apresentando $\mathrm{R}_{\text {ajustado }}^{2}=0,8753$ e $S_{y x}=0,7654$, e $C V=23,59 \%$ por não apresentar tendências de superestimar ou subestimar os volumes dentro da amplitude de diâmetros observados, conforme Figura 1.

As demais equaçóes testadas apresentaram coeficientes de determinaçáo ajustado que variaram de 0,8679 a 0,8774 e erro padrão de estimativa entre 0,7591 a 0,7878 que são valores muitos próximos à equação de Husch. No entanto, a distribuição gráfica dos resíduos apresentou uma variação em escala ligeiramente superior em relação ao gráfico de Husch.

\section{MORTALIDADE E INGRESSO}

A mortalidade registrada no período avaliado foi de 8 indivíduos.ha ${ }^{-1} \cdot$ ano $^{-1}$, com taxa de $0,78 \%$. A taxa média de mortalidade para a área estudada é compatível com estudos realizados na Amazônia por outros pesquisadores, tais como: Higuchi et al. (2004) encontraram 0,7\% para um período de 1986 a 2000 em uma Floresta primária da Amazônia Central; Rocha (2001) pesquisando as taxas de recrutamento e mortalidade para o período de 1996 a 2001 em dois transectos da Floresta de Terra Firme da bacia do Rio Cueiras encontrou taxa de mortalidade de $0,86 \%$.

$\mathrm{O}$ ingresso encontrado na área de estudo foi de 3,49 indivíduos.ha ${ }^{-1} \cdot$ ano $^{-1}$, com taxa de $0,30 \%$. Rocha (2001) pesquisando as taxas de recrutamento e mortalidade para o período 1996 - 2001 em dois transectos da Floresta de Terra

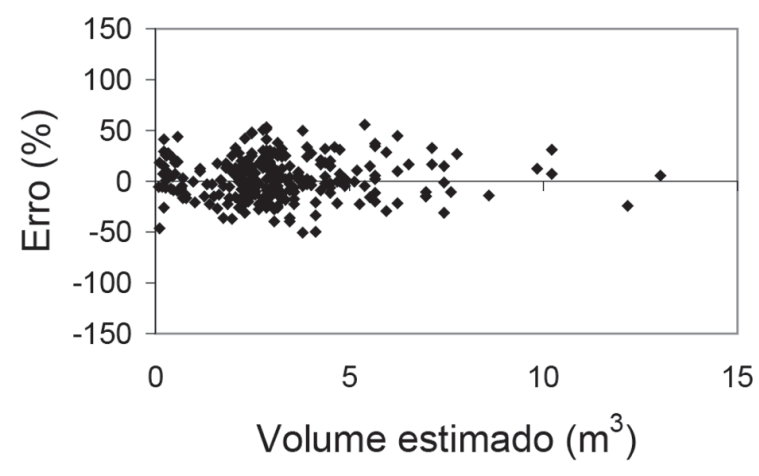

Figura 1 - Resíduos em função do volume estimado para o modelo volumétrico com casca de Husch. 
Firme da bacia do Rio Cueiras encontrou taxa de recrutamento de $0,90 \%$, resultado superior ao ora apresentado na área de estudo. No estudo realizado por Higuchi et al. (2004) também encontrou resultado superior, ao da área deste estudo, com 0,7\% de recrutamento para o período de 1986 a 2000 em uma Floresta primária da Amazônia Central.

\section{ABUNDÂNCIA, INCREMENTO EM DIÂMETRO ÁREA BASAL E VOLUME}

As estimativas médias por hectare de número de indivíduos para a floresta estudada é de 136. Resultados semelhantes para área foram os encontrados por Ribeiro et al. (1999) estudando duas florestas uma em Carajas e outra em Marabá - PA, que consideraram um DAP mínimo de $20 \mathrm{~cm}$, e encontraram uma abundância média para a primeira de 132 indivíduos.ha ${ }^{-1} \mathrm{e}$ para e 128 indivíduos.ha ${ }^{-1}$ para a segunda.

Os gêneros com maiores representantes na área são: Mouriri (14 indivíduos.ha $\left.\mathrm{a}^{-1}\right)$; Ocotea (11 indivíduos.ha ${ }^{-1}$ ) e Manilkara (10 indivíduos.ha ${ }^{-1)}$. Vieira (2003), citada acima, descreve em seu trabalho que as espécies mais abundantes em Manaus foram as do gênero Eschweilera com 63 indivíduos.ha ${ }^{-1}$; em Rio Branco as espécies mais abundantes foram Carapa guianensis (53 indivíduos. $\mathrm{ha}^{-1}$ ), as espécies do gênero Sclerolobium (26 indivíduos. ha ${ }^{-1}$ ) e as do gênero Bauhinia e Rinoreocarpus cada uma delas com 15 indivíduos.ha ${ }^{-1}$.

O diâmetro, a área basal e o volume, bem como seus incrementos periódicos anuais avaliados para a área estudada estáo apresentados na Tabela 3.

Os resultados apresentados na Tabela 3 para a variável área basal na área estudada são inferiores quando comparados aos de Higuchi et al. (1998), Ribeiro et al. (1999), Vidal et al. (2002), Vieira (2003), Mendonça (2003) e Teixeira et al. (2007) pois com exceção do estudo de Mendonça (2003) que avaliou indivíduos com o diâmetro mínimo de $15 \mathrm{~cm}$, os outros estudos avaliaram indivíduos com diâmetro mínimo acima de $10 \mathrm{~cm}$.

Higuchi et al. (1998) e Mendonça (2003) em seus trabalhos encontram valores mais elevados em relação aos resultados expostos acima para a variável volume. O primeiro estudando na regiấo da Bacia do Rio Cuieras encontrou 438 $\mathrm{m}^{3} \cdot \mathrm{ha}^{-1}$ para o transecto (1) e $423 \mathrm{~m}^{3} \cdot \mathrm{ha}^{-1}$ para o transceto
(2) e o segundo em uma área em Itacoatiara medida antes da exploraçáo encontrou $305,6 \mathrm{~m}^{3} \cdot \mathrm{ha}^{-1}$. Teixeira et al. (2007) também encontraram valores médios elevados: $323,97 \mathrm{~m}^{3} \cdot \mathrm{ha}^{-1}$ para o ano de 2000 e 346,38 $\mathrm{m}^{3}$.ha ${ }^{-1}$ para o ano de 2004.

Vidal et al. (2002), na Amazonia Oriental (Paragominas) avaliando indivíduos com diâmetro mínimo acima de $10 \mathrm{~cm}$, na área testemunha, encontraram resultado de incremento diamétrico compatível ao ora estudado, $0,33 \mathrm{~cm} \cdot \mathrm{ano}^{-1}$. Vieira (2003) encontrou resultados parecidos na regiáo de Rio Branco e Santarém, $0,39 \mathrm{~cm} \cdot \mathrm{ano}^{-1}$ e $0,31 \mathrm{~cm} \cdot \mathrm{ano}^{-1}$ respectivamente, considerando indivíduos com diâmetro mínimo acima de $10 \mathrm{~cm}$; no entanto em Manaus encontrou valor bem abaixo aos demais $0,172 \mathrm{~cm} \cdot$ ano $^{-1}$. Resultados também abaixo aos encontrados neste estudo foram expostos por Carvalho (1992), Gomide (1999) e Higuchi et al. (2004). O primeiro trabalhando na Floresta Nacional do Tapajós, Santarém, encontrou incremento de $0,2 \mathrm{~cm} \cdot$ ano $^{-1}$ para uma área onde não houve colheita de madeira; o segundo pesquisando no Projeto Jarí, Amapá, obteve IP de 0,14 cm.ano ${ }^{-1}$ para uma Floresta primária para indivíduos com diâmetro mínimo acima de 5 $\mathrm{cm}$ o terceiro analisando a dinâmica de uma floresta primária da Amazônia Central com indivíduos de diâmetro mínimo acima de $10 \mathrm{~cm}$ obteve IPA de $0,164 \mathrm{~cm}$.

Resultado semelhante ao crescimento em diâmetro foi revelado por Carvalho (1992) trabalhando na Floresta Nacional do Tapajós, onde encontrou $0,4 \mathrm{~cm} \cdot \mathrm{ano}^{-1}$ para uma área com manejo. No estudo em Paragominas Vidal et al. (2002) analisando uma área submetida a procedimentos de manejo obteve $0,63 \mathrm{~cm} \cdot$ ano $^{-1}$, praticamente o dobro do resultado obtido neste trabalho.

Os resultados dos incrementos periódicos anuais (IPA) em área basal da área estudada são superiores ao encontrado por Oliveira (2005) que trabalhando na Floresta Nacional do Tapajós, Belterra, encontrou incremento de $0,06 \mathrm{~m}^{2} \cdot \mathrm{ha}^{-1} \cdot$ ano $^{-1}$ para uma área sem intervençāo.

Teixeira et al. (2007) em uma floresta natural de Terrafirme, na regiâo de Manaus encontrou incremento em área basal superior ao estudo ora apresentado: $0,44 \mathrm{~m}^{2} \cdot \mathrm{ha}^{-1} \cdot$ ano $^{-1}$.

Oliveira (2005) encontrou resultados inferiores de incrementos periódicos anuais de volume para a área sem intervenção em relaçáo a esse estudo: $0,70 \mathrm{~m}^{3} \cdot \mathrm{ha}^{-1} \cdot \mathrm{ano}^{-1}$.

Tabela 3 - Diâmetro, área basal, volume e incremento periódico anual (IPA) encontrados no período estudado para o total de indivíduos em Marcelândia-MT.

\begin{tabular}{|c|c|c|c|}
\hline \multirow{2}{*}{ Variáveis } & \multicolumn{2}{|c|}{ Ano } & \multirow{2}{*}{ IPA (2001-2007) } \\
\hline & 2001 & 2007 & \\
\hline Diâmetro médio $(\mathrm{cm})$ & 32,62 & 34,66 & 0,34 \\
\hline Área basal $\left(m^{2} \cdot h^{-1}\right)$ & 12,12 & 13,45 & 0,22 \\
\hline Volume $\left(\mathrm{m}^{3} \cdot \mathrm{ha}^{-1}\right)$ & 110,83 & 123,50 & 2,11 \\
\hline
\end{tabular}


Teixeira et al. (2007) encontrou incremento em volume de 5,60 $\mathrm{m}^{3} \cdot \mathrm{ha}^{-1} \cdot$ ano $^{-1}$ para uma floresta natural de Terra-firme, na regiáo de Manaus, valor bem superior ao encontrado neste estudo.

\section{CONCLUSÕES}

A metodologia adotada permitiu determinar os incrementos periódicos em diâmetro, área basal, volume e, também, as taxas de mortalidade e ingresso da área estudada, estando estas dentro dos limites aceitáveis para a Amazônia.

A aplicação de técnicas de manejo tornou a floresta mais produtiva uma vez que o crescimento e incremento na área manejada foi maior que em uma área intacta e sem manejo.

\section{BIBLIOGRAFIA CITADA}

ASSMANN, E. 1970. The principles of forest yield study. Pergamon Press, Oxford. 506 pp.

AZEVEDO, C.P. 2006. Dynamics of forests under management in the Eastern Amazon: experimentation and simulation. Tese de Doutorado, Universidade Federal do Paraná, Curitiba. 236 pp. (in Portuguese)

BRAGA, F.M.S.; REZENDE, A.V. 2007. Arboreal vegetation's dynamic in Catetinho's Gallery Forest, Brasília, DF. Cerne, 13 (2): 138 -148. (in Portuguese, with abstract in English)

BRASIL. 1981. Ministério das Minas e Energia. Departamento Nacional de Produção Mineral. RADAMBRASIL Project: Volume 22 Folha Sc 22 - Tocantins. Rio de Janeiro.

CARVALHO, J.O.P. 1992. Structure and dynamics of a logged over Brazilian Amazonian rain forest. Thesis, Oxford University, Oxford. 236 pp.

CHAPMAN, H.H.; MEYER, W.H. 1949. Forest mensuration. McGuam - Hill Book Company, New York. 522 pp.

DAJOZ, R. 2006. Ecology principles. Artmed, Porto Alegre. 519 pp. (in Portuguese)

D’OLIVEIRA, M.V.N.; BRAZ, E.M. 2006. Forest dynamics study of the managed forest of the PC Peixoto Communit forest management project in western Amazon. Acta Amazonica, 36 (2):177-182. (in Portuguese, with abstract in English)

DRAPER, N.; SMITH, H. 1998. Applied regression analysis. John Wiley \& Sons, New York. 736 pp.

GOMIDE, G.L.A. 1999. Structure and dynamics of growth of tropical forests primary and secondary in the State of the Amapá. In: SIMPÓSIO SILVICULTURA NA AMAZÔNIA ORIENTAL: Contribuições do Projeto EMBRAPA/DFID, 1999, Belém, Anais... Belém: EMBRAPA. p.195-202.

HIGUCHI, N.; CHAMBERS, J.Q.; SANTOS, J.; RIBEIRO, R.J.; PINTO, A.C.M.; SILVA, R.P.; ROCHA, R.M.; TRIBUZY, E.S. 2004. Carbon balance and dynamics of primary vegetation in the Central Amazon. Floresta, 34 (3): 377-384. (in Portuguese, with abstract in English)HIGUCHI, N.; CAMPOS, M.A.A.;
SAMPAIO, P.T.B.; SANTOS, J. 1998. Forest research for the conservation of the forest and Mine rehabilitation of the Amazonia. INPA, Manaus. 264pp. (in Portuguese)

HOSOKAWA, R.T.; MOURA, J.B.; CUNHA, U.S. 1998. Introduction to the management and economy of forests. UFPR, Curitiba. 162 pp. (in Portuguese)

JARDIM, F.C.S.; VOLPATO, M.M.L.; SOUZA, A.L. 1993. Dynamics of natural succession in gaps of tropical forests. SIF, Viçosa. 60 pp. (Boletim SIF, 10). (in Portuguese, with abstract in English)

LAMPRECHT, H. 1990. Silviculture in the tropics: tropical forest ecosystems and their tree species: possibilities and methods for their long-term utilization. Eschborn: GTZ, 1990. 343pp. (in Portuguese)

MACHADO, S.A.; URBANO, E.; CONCEIÇÃO, M.B.; FIGUEIREDO FILHO, A.; FIGUEIREDO, D.J. 2004. Comparison of taper functions for different ages and thinning regimes in Pinus oocarpa plantations. Boletim de Pesquisa Florestal, (48): 41-64. (in Portuguese, with abstract in English)

MENDONÇA, A.C.A. 2003. Characterization and simulation of dynamic processes of a tropical forest of "terra-firme" using the Transition Matrix simulation technique. Dissertação de Mestrado, Universidade Federal do Paraná, Curitiba. 92 pp. (in Portuguese, with abstract in English)

OLIVEIRA, L.C. 2005. Effect of logging and thinning intensities on the dynamic of vegetation of an area of 136 ha in the Tapajos National Forest. Tese de Doutorado, Escola Superior de Agricultura Luiz de Queiroz, Piracicaba. 183 pp. (in Portuguese, with abstract in English)

PÉLLICO NETO, S.; BRENA, D.A. 1997. Forest inventory. Curitiba. 316pp. (in Portuguese)

PRODAN, M. 1968. Forest biometrics. Pergamon Press, Oxford. $447 \mathrm{pp}$.

RIBEIRO, R.J.; HIGUCHI, N.; SANTOS, J.; AZEVEDO, C.P. 1999. Plant structural analysis on Carajás and Marabá Regions - Para State, Brazil. Acta Amazônica, 29 (2) : 207-222. (in Portuguese, with abstract in English)

ROCHA, R.M. 2001. Ingrowth and mortality rates of Terra-firme forest of Rio Cuieiras basin in Manaus-AM region. Dissertação de Mestrado, Universidade do Amazonas, Manaus. 49 pp. (in Portuguese, with abstract in English)

TEIXEIRA, L.M.; CHAMBERS, J.Q.; SILVA, A.R.; LIMA, A.J.N.; CARNEIRO, V.M.C.; SANTOS, J.; HIGUCHI, N. 2007. Dynamics of the Terra-firme primary forest in Manaus-AM region using the Markov probabilistic transition matrix. Acta Amazonica, 37 (3): 377-384. (in Portuguese, with abstract in English)

VANCLAY, J.K. 1994. Modelling forest growth and yield. CAB Internacional, Wallingford. $312 \mathrm{pp}$.

VIDAL, E.; VIANA, V.M.; BATISTA, J.L.F. 2002. Regrowth of a tropical rain forest in Eastern Amazonia three years after planned and unplanned logging. Scientia Forestalis, (61): 133 -143. (in Portuguese, with abstract in English) 


\section{ACTA}

VIEIRA, S.A. 2003. Global changes and tree growth rate in the Amazon forest. Tese de Doutorado, Escola Superior de Agricultura Luiz de Queiroz, Piracicaba. 133 pp. (in Portuguese, with abstract in English)

Recebido em 04/12/2008

Aceito em 21/02/2010 